\section{MS15-P15 Study and Characterization of a Composite for Use in the Automotive Industry with the Use of X-ray Powder Diffraction}

Kelly C.L. Lixandrão ${ }^{1}$

\section{Federal University of $\mathrm{ABC}$}

email: kellycrislira@yahoo.com.br

The development of the automotive industry in the twentieth century has increased the production of motor vehicles, generating an increase of the national fleet, and the consumption of tires reached staggering numbers. By this reason, the amount of solid wastes generated and deposited in the environment has grown considerably. In order to minimize the impacts arising from this deposition, some environmental agencies have created resolutions to ensure proper disposal of scrap materials. [1]. At the end of its service life, the wasted tires, without shooting conditions or reforms, are difficult to recycle because the vulcanization process makes the material infusible and of difficult processability. When discarded in inappropriate locations, tires may serve as a medium for the development of disease vectors besides representing risk of fire, polluting the air with toxic fumes - containing pollutants such as carbon and sulfur - as well as releasing oil, which can contaminate the water table [2]. In order to reduce the amount sent to the environment and to generate an appropriate production cycle, the wasted tire was used as reinforcement in a polymeric matrix to be applied to the engine encapsulation of commercial vehicles. It is intended to provide a reduction of the piece weight and thus the vehicle, optimizing fuel consumption and reducing the emission of pollutants; additionally, it ensures the minimization of noise pollution through the noise attenuation by the composite. Acoustic analysis of transmission loss and external noise were performed to verify whether the material complies with the limits specified by the resolutions. The X-ray powder diffraction analysis was fulfilled to identify and quantify the different phases that composing the material.

Keywords: tire powder, X-ray diffraction, engine encapsulation.

\section{MS15-P16 Characterization of lone electron pair using Liebau density vector and Wang-Liebau eccentricity parameters \\ M. Mangir Murshed ${ }^{1}$, Cecilia B. Mendive ${ }^{2}$, Mariano Curti ${ }^{2}$, Thorsten M. Gesing ${ }^{1}$}

1. Chemische Kristallographie fester Stoffe, Institut für Anorganische Chemie und MAPEX Center for Materials and Processes, Universität Bremen, Leobener Straße /NW2, D-28359 Bremen, Germany

2. Departamento de Química, Facultad de Ciencias Exactas y Naturales, Universidad Nacional de Mar del Plata, Dean Funes 3350, 7600 Mar del Plata, Argentina

\section{email: murshed@uni-bremen.de}

Compounds carrying cations with lone electron pair (LEP) show marked distortion in their coordination sphere, which is intimately related to the electronic distribution of the LEP. Hence, non-spherical but asymmetric LEPs are usually referred to be stereochemically active. It is still in search of a measure to quantify at which extend the LEP of a cation in a crystal structure is stereochemically active. Herein we approach the Wang-Liebau eccentricity (WLE) parameter [1] and the Liebau density vector (LDV) [2] to characterize the $6 s^{2}$ LEP of $\mathrm{Bi}^{3+}$ cations in $\mathrm{Bi}_{4} \mathrm{~B}_{2} \mathrm{O}_{9}$ as a model compound. WLE is a vector term associated with all bonds in a given coordination, which measures the electronic deformation density of the LEP and directs along the line from the nucleus to the approximate center of the LEP charge density. The dimensionless length of the WLE parameter thus indirectly measures the degree of deformation of the associated LEP, and cannot be depicted graphically. LDV describes the length of a vector directed from the nucleus of an atom to the maximum electron density of the LEP. We have demonstrated on some mullite-type compounds that the direction of WLE and LDV are almost parallel as long as a localized electron density is obtained for LDV [2]. The ambient condition crystal structure of $\mathrm{Bi}_{4} \mathrm{~B}_{2} \mathrm{O}_{9}(P 2 / c)$ $[3,4]$ possesses four crystallographically different $\mathrm{Bi}$-positions, where $\mathrm{Bi} 1$ and $\mathrm{Bi} 3$ are six-coordinated and, $\mathrm{Bi} 2$ and $\mathrm{Bi} 4$ are seven and eight coordinated, respectively, within $\mathrm{Bi}-\mathrm{O}$ distance less than $330 \mathrm{pm}$. Both the WLE and LDV magnitudes for Bi1 and $\mathrm{Bi} 3$ are higher than those of the higher coordinated $\mathrm{Bi} 2$ and $\mathrm{Bi} 4$. A correlation between the LDV and the electron density of LEP determines the minimum localized electron density of LEP to be stereochemically active irrespective to the crystallographic position of the $\mathrm{Bi}$-atom. With increasing temperature both the WLE and LDV decreased, indicating the decrease of stereochemical activity of the LEP.

References: [1] X. Wang, F. Liebau, Z. Kristallogr. 211 (1996) 437. [2] M. Curti, Th.M. Gesing, M.M. Murshed, T. Bredow, C.B. Mendive, Z. Kristallogr. 228 (2013) 629. [3] S.K. Filatov, Y.F. Shepelev, Y.V. Aleksandrova, R.S. Bubnova, Russ. J. Inorg. Chem. 52 (2007) 21. [4] A. Hyman, A. Perloff, Acta Crystallogr. 28 (1972) 2007.

Keywords: lone electron pair, Liebau density vector, Wang-Liebau eccentricity 